\title{
Overview of the effects of chemical mixtures with endocrine disrupting activity in the context of real-life risk simulation (RLRS): An integrative approach (Review)
}

\author{
DENISA MARGINA ${ }^{1}$, GEORGE MIHAI NIȚULESCU ${ }^{1}$, ANCA UNGURIANU ${ }^{1}$, \\ ROBIN MESNAGE ${ }^{2}$, MARINA GOUMENOU ${ }^{3}$, DIMOSTHENIS A. SARIGIANNIS ${ }^{4-6}$, MICHAEL ASCHNER ${ }^{7}$, \\ DEMETRIOS A. SPANDIDOS ${ }^{8}$, ELISAVET A. RENIERI ${ }^{9}$, ANTONIO F. HERNÁNDEZ ${ }^{10}$ and ARISTIDIS TSATSAKIS ${ }^{3}$ \\ ${ }^{1}$ 'Carol Davila’ University of Medicine and Pharmacy, 020956 Bucharest, Romania; ${ }^{2}$ Gene Expression and Therapy Group, \\ Department of Medical and Molecular Genetics, Faculty of Life Sciences and Medicine, King's College London, \\ London SE1 9RT, United Kingdom; ${ }^{3}$ Department of Forensic Sciences and Toxicology, Faculty of Medicine, \\ University of Crete, 71409 Heraklion; ${ }^{4}$ Department of Chemical Engineering, Environmental Engineering Laboratory, \\ Aristotle University of Thessaloniki, 54124 Thessaloniki; ${ }^{5}$ HERACLES Research Center on the Exposome and Health, \\ Center for Interdisciplinary Research and Innovation, Balkan Center, 57001 Thessaloniki, Greece; \\ ${ }^{6}$ Environmental Health Engineering, Department of Science, Technology and Society, School for Advanced Study (IUSS), \\ 27100 Pavia, Italy; ${ }^{7}$ Department of Molecular Pharmacology, Albert Einstein College of Medicine, Bronx, \\ NY 10463, USA; ${ }^{8}$ Laboratory of Clinical Virology, and ${ }^{9}$ Centre of Toxicology Science and Research, \\ School of Medicine, University of Crete, 71409 Heraklion, Greece; ${ }^{10}$ Department of Legal Medicine \\ and Toxicology, University of Granada School of Medicine, Granada, Spain
}

Received June 11, 2019; Accepted August 2, 2019

DOI: 10.3892/wasj.2019.17

\begin{abstract}
Research over the past years has indicated that chronic human exposure to very low doses of various chemical species in mixtures and administered via different routes (percutaneous, orally, etc.) should be the main focus of new biochemical and toxicological studies. Humans have daily contact with various chemicals, such as food additives, pesticides from fruits/vegetables, antibiotics (and other veterinary drugs) from meat, different types of preservatives from cosmetics, to name a few. Simultaneous exposure to this wide array of chemicals does not produce immediate effects, but summative effect/s over time that may be clinically manifested several years thereafter. Classical animal studies designed to test the toxic outcome of a single chemical are not suitable to assess, and then extrapolate to humans, the effects of a whole mixture of chemicals. Testing the aftermath of a combination of chemicals, at low doses, around or below the no observed
\end{abstract}

Correspondence to: Professor Aristidis Tsatsakis, Department of Forensic Sciences and Toxicology, Faculty of Medicine, University of Crete, Voutes, 71409 Heraklion, Greece

E-mail: aristsatsakis@gmail.com

Key words: real-life risk simulation, chemical mixtures, endocrine disruptors adverse effect is stressed by many toxicologists. Thus, there is a need to reformulate the design of biochemical and toxicological studies in order to perform real-life risk simulation. This review discuss the potential use of computational methods as a complementary tool for in vitro and in vivo toxicity tests with a high predictive potential that could contribute to reduce animal testing, cost and time, when assessing the effects of chemical combinations. This review focused on the use of these methods to predict the potential endocrine disrupting activity of a mixture of chemicals.

\section{Contents}

1. The need for a change in the current scientific paradigm

2. Relevance of exposure to mixtures of endocrine disruptor chemicals in the context of 21st century research

3. Future directions in real-life risk simulations of endocrine disruptors

4. Conclusions

1. The need for a change in the current scientific paradigm

Human populations come into contact, on a daily basis, with a large range of chemical mixtures, at low levels of exposure, from virtually every product that is used, from the food consumed (raw or processed, either vegetal, containing soil originating substances, or animal), drinking water (tap or bottled), the air 
that is breathed, consumer products (cosmetics, either artisanal or industrial), etc. The results from a nation-wide survey of environmental contaminants among 4,145 pregnant women in France in 2011 indicated that bisphenol A, phthalates, pesticides (such as pyrethroids), dioxins, furans, polychlorobiphenyls, brominated flame retardants, perfluorinated compounds and heavy metals are quantifiable in virtually all individuals (1). Over the past 15 years, researchers from different disciplines (toxicologists, biochemists, chemists, medical doctors and molecular biologists) have made constant efforts to unravel the possible effects induced by the long-term exposure to low doses of chemicals on human physiology (2) (Fig. 1).

The realization that humans are exposed to a large number of substances through different routes in their everyday lives has led to a change in scientific paradigms (2). To date, the majority of studies, and in particular regulatory toxicology studies, have focused on testing multiple outcomes resulting from the administration of a single substance at medium-high doses to laboratory animals (3). Also, biomonitoring studies (carried out mostly in urine samples) have confirmed exposure to different chemicals. Although the presence of chemicals (or their metabolites) in biological samples does not constitute a proof of the occurrence of adverse health effects in humans, it represents a source of concern. However, there is growing evidence of the effects of chemical mixtures at concentrations for which individual components failed to elicit adverse effects when tested individually (4,5) (Fig. 2).

Data on the effects of chemical mixtures are increasingly taken into consideration by the scientific community and regulatory agencies worldwide to issue regulations and guidelines to understand better the long-term effects of exposure to environmental (or dietary) mixtures of chemicals in real-life exposure scenarios and to protect/preserve the human health. Although humans are not exposed simultaneously to all existing chemicals; the assessment of an infinite number of potential chemical combinations in not feasible from a regulatory point of view. Hence, the most representative chemical mixtures, and their risk drivers, should be identified at first, and then validated and internationally accepted tools can be applied to assess their potential combined effects.

Toxicological studies testing combinations of chemicals at low doses, around or below their no observed adverse effect level (NOAEL), mimicking real-life scenarios, under the framework of real-life risk simulation (RLRS), are imperative to evaluate the effects induced by these chemical mixtures in humans (6-13).

Another area of research for future studies investigating the effects of mixtures of environmental pollutants is the role of the gut microbiome. Communities of microorganisms inhabiting the human gastrointestinal tract have the ability to metabolize a large range of chemicals and affect their therapeutic efficacy or their toxicity (14). Since the potential chemical metabolism of the gut microbiome remains largely uncharacterized, recent reviews have encouraged the conduct of studies simulating real-life exposure to mixtures using laboratory animals or simulators of the human gut microbiome ecosystem (15). Machine learning algorithms could also help developing reliable approaches to simulate gut microbiome metabolism and its consequence on human health in future studies (16).

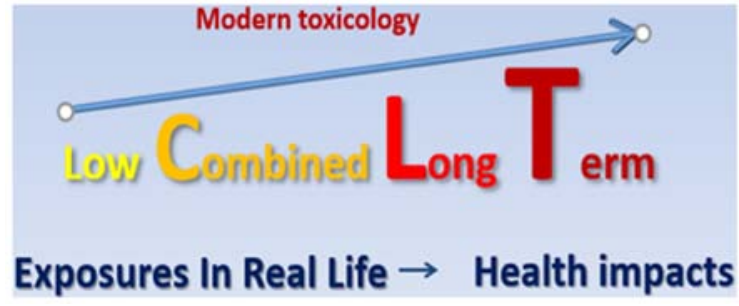

Figure 1. Paradigm change in the toxicological study design.

\section{Relevance of exposure to mixtures of endocrine disruptor chemicals in the context of 21 st century research}

One of the most important areas of concern regards the potential health effects of exposure to low doses of mixtures of endocrine disruptors (EDs) also known as endocrine-disrupting chemicals (EDCs). Modern lifestyles result in ubiquitous daily exposures to a combination of environmental mixtures of EDs that can accumulate in the body tissues and fluids. Human exposure, particularly at very low-doses, is continuous and occurs in different mixtures with potential effects that may not be predictable when evaluating individual compounds. Thus, the assessment of potential human risks resulting from exposure to mixtures of EDs is crucial for consumer safety (17). Moreover, recent evidence indicates that exposure to these chemicals during development can affect not only the exposed individuals, but also their offspring and future generations as a result of epigenetic modifications (18).

Specifically, synthetic compounds can contain polycyclic aromatic structures, resembling the structure of endogenous hormones. By interacting on specific receptors, and depending on their concentration, affinity and potency, they can elicit effects by mimicking natural hormones. For this reason, such chemicals can also exert effects even at very low concentrations (e.g., steroid hormones, such as dehydroepiandrosterone sulphate (DHEAS) can have effects at femtomolar concentrations) (19). By interfering with physiological endogenous systems, EDs impair the hormone balance and disrupt normal function, ultimately inducing toxicological effects. Exposure to such substances is of particular concern in sensitive periods, such as the prenatal period, as these exposures can lead to irreversible changes in the developing organs and increase the susceptibility to develop diseases later in life. Nevertheless, there is still controversy concerning the possible role of exposure at real-life concentrations to environmental chemicals and certain endocrine-related human diseases, such as hormone-related cancers, reproductive disorders, obesity, diabetes and neurodevelopment disorders (20). Certainly, EDs interfere with brain development through changes in thyroid hormone levels that are essential for the development of the nervous system.

Different International Organizations and Agencies have provided a similar definition for EDs. WHO defines an ED as 'an exogenous substance or mixture that alters function(s) of the endocrine system and consequently causes adverse health effects in an intact organism, or its progeny, or (sub)populations' (21). This is also the working definition adopted by the European Commission (EC) (22). The European Food Safety Authority (EFSA) inserted the term 'Endocrine Active Substances (EASs)' defined as 'any chemical that can interact directly or indirectly 


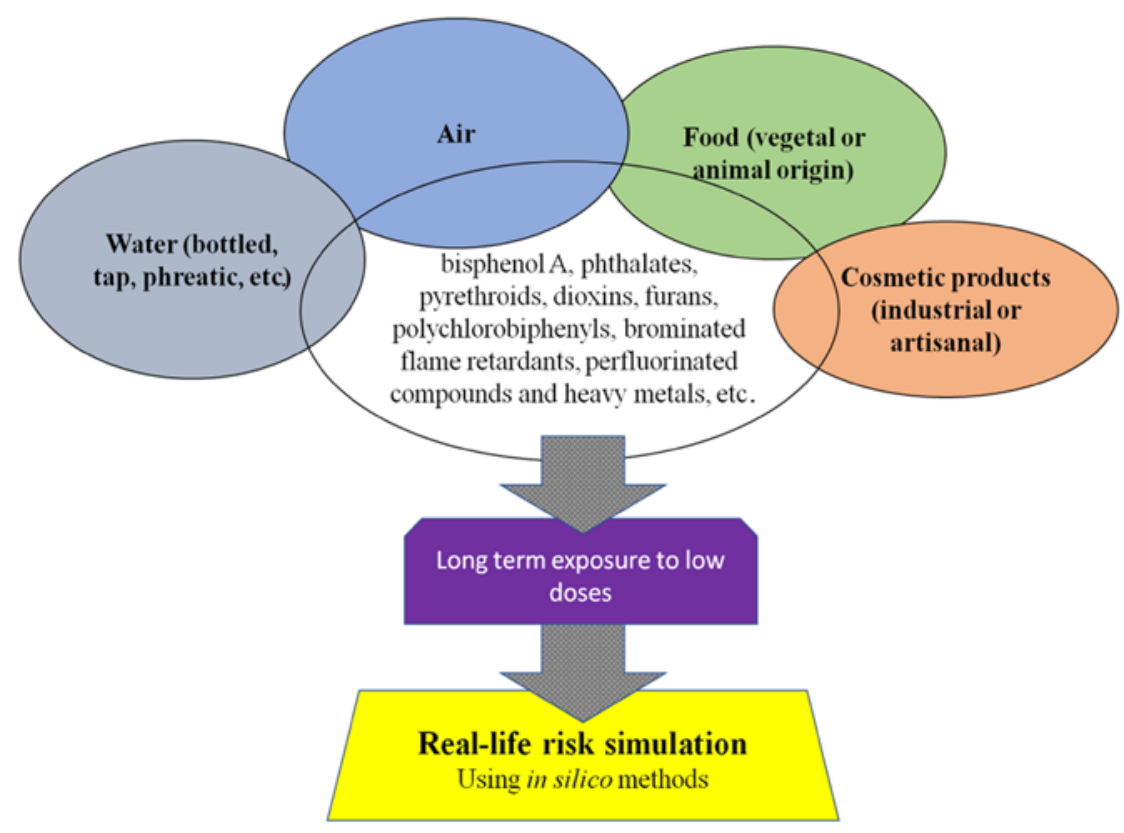

Figure 2. Low-dose chronic exposure to chemical mixtures: An important issue in contemporary studies.

with the endocrine system, and subsequently result in an effect on the endocrine system, target organs and tissues' (23). The reason for inserting this term was to discriminate between chemicals that may interfere with the endocrine or hormone systems without inducing adverse outcomes.

The Environmental Protection Agency (US-EPA) defined EDs as "exogenous agents that interfere with the production, release, transport, metabolism, binding, action, or elimination of the natural hormones in the body responsible for the maintenance of homeostasis and the regulation of developmental processes' (24). EDs have been linked from fertility disturbances to a number of highly prevalent human pathologies, such as obesity, cancer and diabetes mellitus (23-32). Trasande et al (2015) estimated that EDs contribute at least $€ 157$ billion per year to the cost of human disease in the European Union (EU) (33). In the US, the estimated figure is even larger, reaching $\$ 340$ billion per annum (34).

There are some points to be considered when discussing EDs in the context of RLRS. Firstly, there is the incredible chemical diversity of EDs. These can include natural substances from plants and/or fungi (such as phytoestrogens), pharmacologically active molecules (such as contraceptive hormones or molecules used in hormone-responsive malignancies), chemicals used as additives, preservatives in food/cosmetics, pesticides, solvents, lubricants, fungicides and other types. Chemical structures also vary considerably, some of them being clustered based on their common structure, such as polychlorinated derivatives, bisphenols, dioxins, phthalates, or diethylstilbestrol $(35,36)$.

Existing assays are currently focused on the estrogen, androgen, thyroid and steroidogenesis (EATS) pathways and less on non-EATS modalities. However, standard chronic apical toxicity tests are capable of detecting most downstream effects of perturbation of the non-EATS pathways (20).

A useful toxicological tool for EDs is the Endocrine Disruptor Knowledge Base (EDKB), an online library available at the US FDA, containing experimental data for
$>3,200$ chemical compounds and serves as a resource for both research and regulatory scientists (37). Based on the EDKB, the National Center for Toxicological Research (NCTR) of the US is currently developing methods and models for the computational prediction of endocrine-related risks.

Similarly, the Endocrine Active Substances Information System (EASIS) was developed in the EU. EASIS can be used to search for results from scientific studies on chemicals related to endocrine activity. Currently, it contains information on $>500$ different chemicals based on in vitro and in vivo assays in various species. However, the presence of a substance in the database does not mean necessarily that it is an ED. A new and improved version, EASIS 2.0, is anticipated to be published soon (38).

The Organization for Economic Cooperation and Development (OECD), in 2018, updated the document entitled 'Revised Guidance Document 150 on Standardized Test Guidelines for Evaluating Chemicals for Endocrine Disruption' as a standard for the assay to be used for the identification of new EDs based on endocrine signaling pathways (39). When data are lacking, the document advises the use of quantitative structure-activity relationship (QSAR) models, analogue, category and read-across approaches for hazard identification. On its website, the OECD made available a free QSAR Toolbox that can be used as standalone software or for a better interpretation of the mechanisms underlying in vivo results.

The EC requested EFSA and ECHA to develop a common harmonized guidance to ensure that the endocrine disruptor criteria adopted by the EU in 2017 are applied consistently for the assessment of biocides and pesticides. For drafting this guidance, the Joint Research Centre (JRC), the EC's science and knowledge service provided its support due to its expertise in the area and previous reports development (23).

The US EPA has a dedicated Endocrine Disruptor Screening Program (EDSP) to identify substances that have the potential to interact with the estrogen, androgen, or thyroid 
hormone systems and to establish a dose-effect relationship. The program uses two major exposure models, the first being 'off-the-shelf' chemicals released into the environment by the industry, and the second concerns consumer and in-home chemicals ingredients (40). Recent studies have shown that the ToxCast database can be profitably used to elucidate the mechanisms of action of chemicals acting as obesogens, such as neonicotinoids (41), or as estrogen receptor agonists, such as bisphenol A alternatives (42).

\section{Future directions in real-life risk simulations of EDs}

EDs mixtures used in experimental studies are very simple and consist of unrealistic mixtures compared to the real-world scenario. As such, the net effect in humans of a mixture of numerous EDs with diverse activities is unpredictable and requires further developments. Computational methods are an essential tool in the drug discovery process, and they are intensively used for the identification of new EDs, considering the time and cost consuming efforts to test all household and industrial chemical ingredients. Computational methods are an important complementary tool for in vitro and in vivo toxicity tests with a high predictive potential that can contribute to identify and assessing risks, and ultimately to reduce animal testing, cost and time (43). The application of machine learning methods on toxicological 'big data' has already been shown to outperform animal test reproducibility (36). This has also been proven to be a successful strategy for determining the effects of chemical mixtures, such as those comprised by EDs. For example, a recent study identified that both the pharmacological estrogen, $17 \alpha$-ethinylestradiol, and the pesticide, trans-Nonachlor, were not able to activate the pregnane $\mathrm{X}$ receptor (PXR) individually; however, when combined, they were efficacious. A biophysical analysis complemented by structural bioinformatics analysis revealed that these compounds formed supramolecular ligands, allowing the combined chemical structure to fit into and activate the ligand binding pocket of the PXR (44).

There is a wide range of computational models, varying from read across, chemical categories, absorption, distribution, metabolism, and elimination (ADME) predictive models, physiologically-based pharmacokinetic (PBPK) models, quantitative structure activity relationships (QSARs), docking and molecular dynamics that are currently used to identify new EDs and to predict their mechanisms of action (45). The predictive power of these methods depends on their selectivity and specificity (46). For example, some studies have suggested that molecular docking methods are not the best choice to evaluate androgen receptor antagonists, while the results of QSAR analyses and molecular dynamics simulations have acceptable sensitivities and specificities $(46,47)$. Kar et al developed a QSAR model to evaluate mixtures of perfluoroalkyl substances (PFASs), an important class of ED pollutants, based on zebrafish embryos development data. The predicted chemicals mixtures displayed a concentration addition pattern suggesting a similar mode of toxic action and non-interaction (48). In the case of estrogen receptors, a large range of methods has been applied in large-scale modeling projects (49). Molecular dynamics simulations have also been used to study the interaction between glyphosate and estrogen receptor alpha (42). It is crucial that researchers and all regulatory agencies understand the draw- backs, limitations and confidence limits of each method. As an example, pharmacophore models work very well for estrogen and androgen receptors, although they are limited in the case of various enzymes that control hormone metabolism $(50,51)$.

There is no single tool available with which to identify all types of potentially active groups; thus, several methods need to be used. The accumulation of biological data from several types of assays on EDs will increase the accuracy of the computational models and will certainly expand their usefulness. In addition, with 48 nuclear hormone receptors (52), many more peptide receptors and an unknown number of signaling pathways as potential targets for these chemicals, the conceivable effects on human biological pathways is massive.

Another important point is the difficulty encountered in the extrapolation of the effects induced by the EDs from an in vivo tested dose to a RLRS model, based on the fact that the dose-response curves for such chemicals are under an intense debate $(53,54)$. As an example, for a number of years, the regulation of pesticides has been based on the paradigm that larger doses (above the NOAEL) result in larger effects, i.e., "the dose makes the poison'. However, studies published over the last 15 years have demonstrated toxic effects of combinations of chemicals at concentrations lower than the NOAEL that can disrupt biological systems (55), suggesting that this area requires further scrutiny (56). The cumulative risk assessment of chemicals in mixtures should be considered in addition to the evaluation of their individual effects (57). Another area of interest that represents a relevant challenge to human health is the non-monotonic dose-response relationships (NMDR). Under this hypothesis, the effects at low doses cannot be predicted from effects at high doses and, if confirmed, chemical testing would need to be changed to protect human health. One review of 51 studies identified 170 non-monotonic dose-response relationships (58); nevertheless, the majority of data comes from in vitro studies.

Evaluating the effects induced by mixtures of chemicals, only considering the ED class, can be very challenging due to the multitude of possible complex combinations of chemicals/chemical classes that humans can come in contact with. Sarigiannis et al proposed a comprehensive framework for addressing this challenge (59). The identification of an effective model to test the above-mentioned effects, is the first step in this type of research. There are, of course, two criteria to be met: to find the mixture of molecules that mimics best real-life situations and to find a way to evaluate the effects induced in vivo. Since the doses are low, the realization of chronic feeding studies is preferable, but complex and costly. In this context, molecular modeling may constitute a solid first step in such an endeavor.

It is practically impossible to test all the combinations of EDs, even for binary mixtures. The groundbreaking work of Bliss identified three categories of joint action in mixtures (60). In the first pattern, the combined effect is the sum of the components, their toxic effect being independent, even if the toxicological mechanism is the same or not. It is the simplest case, as the proportions of each component do not alter their combined effect. In the second case, the toxic effect is not independent and it can be greater than that of each constituent in the case of a synergistic action or lower in the case of antagonistic effects. In the particular case of ED mixtures, the synergistic 
1. Define the mixture

2. Develop HI method

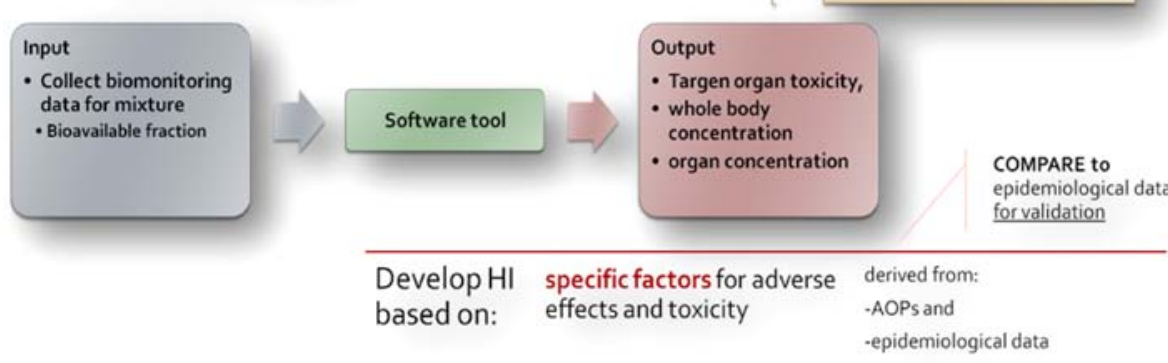

\section{Compare to the $\mathrm{HI}$ approach used up to date}

Figure 3. Exposure assessment to real mixtures and HI estimation method: Fit for purpose. HI, hazard index.

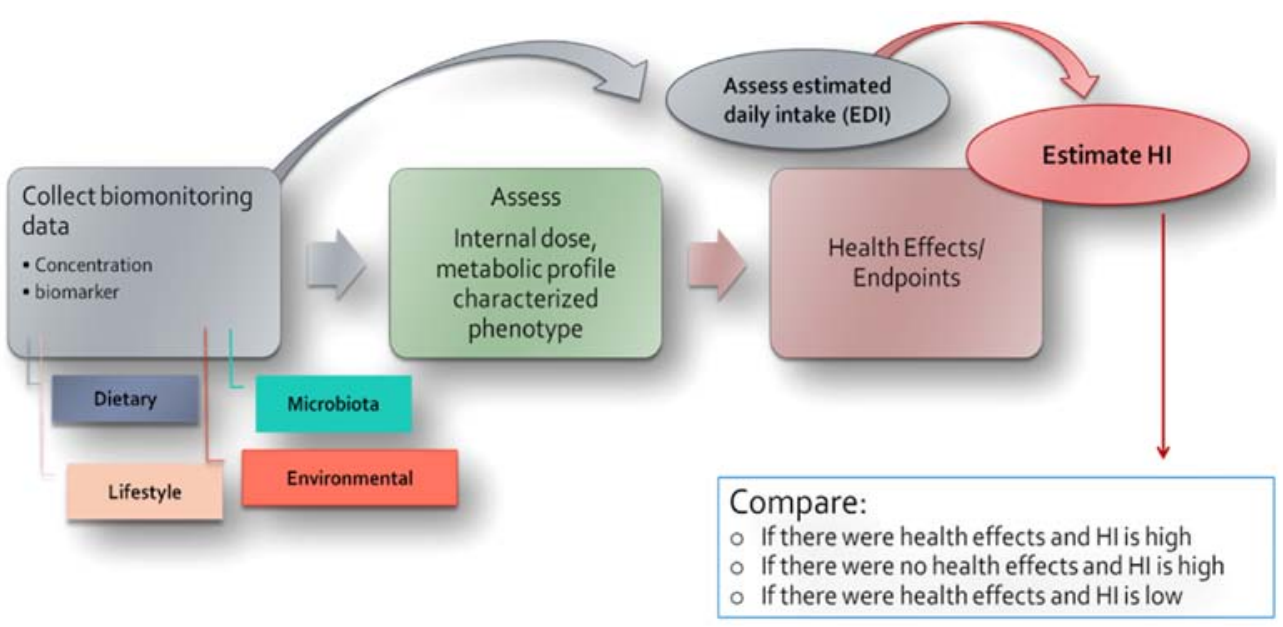

Figure 4. Extensions of the internal dose approach.

action is the most important and several models have been developed to address this problem (61). However, at low exposure levels (around the NOAEL), synergism or antagonism are considered to be unlikely or toxicologically insignificant.

The generalized concentration addition is a mathematical model that evaluates the interaction between mixtures components using a response function independent of the response functions of each individual constituent. The receptor-oriented approach in cumulative risk assessment changes the paradigm from the traditional source-oriented approach, focusing on the exposure assessment of humans to EDs, coupled with effect assessment considering a time variable exposure.

Acknowledging the infinite possible combination of mixtures, the development of hazard estimation approaches fit for purpose, instead of the "umbrella" approaches to cover all grounds, might be more appropriate in certain cases (62). With a view to alleviate uncertainties, a method outline for defined mixtures is proposed (Fig. 3). A three-step process is described, where firstly the mixture in question has to be defined. The mixture (components and portions) is meant to reflect real exposures [e.g., measuring the occurrence and concentration of substances in drinking water of a specific area for the development of a specific hazard index (HI)].

Moving to the second step, the basic notion for the development of the HI is to collect human biomonitoring data on the substances determined and via a software tool, which assesses aggregated (e.g., INTEGRA) or cumulative exposure, to obtain data on certain adverse effects' markers, e.g., biomarkers of target organ toxicity. The data obtained from the in silico model could be integrated with data from other lines of evidence (in vivo, in vitro, epidemiology) concerning the same compounds. This could help i) fill the gaps in describing adverse outcome oathways (AOPs) for the specific mixture and not individual substances; and ii) develop an adversity specific HI which is to be compared with the currently applied HI and evaluated accordingly, as the last step of the proposed process.

Furthermore, aiming to reach more realistic risk characterization methods, an extension of the internal dose approach is proposed (Fig. 4). This approach is based on collecting human biomonitoring data regarding cumulative exposure (dietary, lifestyle, environmental and microbiota) in order to assess the internal dose for the compounds of a specific mixture or characterize the metabolic profile phenotype. Following the internal dose assessment, health effects and toxicity endpoints can be determined. The biomonitoring data could additionally be used for assessing the estimated daily intake (EDI) (63) and subsequently estimating the HI. A comparison between described health effects and the HI estimated could serve as an evaluation of the method (64). 


\section{Conclusions}

Endocrine-mediated adverse effects of chemical mixtures cannot be always identified in standard toxicological studies performed to comply with regulatory requirements. Therefore, supplementary and more focused mechanistic studies may be necessary to further investigate an endocrine mode of action. Despite all limitations, it can be considered that the use of in silico methods to evaluate complex RLRS models will have a great impact and such methods will become a powerful toxicological tool. Those methods can contribute to the identification of potential new EDs and to the prediction of their toxicological targets, thus becoming an effective method to concentrate on similar toxicity pathways and mechanisms of action.

\section{Acknowledgements}

Not applicable.

\section{Funding}

Michael Aschner was supported by National Institute of Health (NIH) R01 ES10563, R01 ES07331 and R01 ES020852. This study was also partially supported by the Special Research Account of University of Crete (ELKE No.4920), the University of Crete spin-off ToxPlus S.A.

\section{Availability of data and materials}

Not applicable.

\section{Authors' contributions}

DM and AU wrote sub-sections 1 and 2. GMN, RM, MG, DASa and EAR contributed to sub-sections 2 and 3. DM, AU, GMN, RM, MG, DASa and EAR also contributed to the search of the literature for this review and to the selection of appropriate references for citation. MA, DASp, AFH and AT performed the critical review of the manuscript and contributed to the conception and design of the study.

\section{Ethics approval and consent to participate}

Not applicable.

\section{Patient consent for publication}

Not applicable.

\section{Competing interests}

The authors declare that they have no competing interests.

\section{References}

1. Dereumeaux C, Saoudi A, Pecheux M, Berat B, de Crouy-Chanel P, Zaros C, Brunel S, Delamaire C, le Tertre A, Lefranc A, et al: Biomarkers of exposure to environmental contaminants in French pregnant women from the Elfe cohort in 2011. Environ Int 97: 56-67, 2016.
2. Tsatsakis A, Goumenou M, Liesivuori J, Dekant W and Hernández AF: Toxicology for real-life risk simulation - Editorial preface to this special issue. Toxicol Lett 309: 33-34, 2019.

3. Kar S and Leszczynski J: Exploration of Computational Approaches to Predict the Toxicity of Chemical Mixtures. Toxics 7: 7, 2019.

4. da Fonseca TG, Abessa DMS and Bebianno MJ: Effects of mixtures of anticancer drugs in the benthic polychaete Nereis diversicolor. Environ Pollut 252 (Pt B): 1180-1192, 2019.

5. Thrupp TJ, Runnalls TJ, Scholze M, Kugathas S, Kortenkamp A and Sumpter JP: The consequences of exposure to mixtures of chemicals: Something from 'nothing' and 'a lot from a little' when fish are exposed to steroid hormones. Sci Total Environ 619-620: 1482-1492, 2018

6. Tsatsakis AM, Docea AO, Calina D, Buga AM, Zlatian O, Gutnikov S, Kostoff RN and Aschner M: Hormetic Neurobehavioral effects of low dose toxic chemical mixtures in real-life risk simulation (RLRS) in rats. Food Chem Toxicol 125: 141-149, 2019.

7. Tsatsakis AM, Docea AO and Tsitsimpikou C: New challenges in risk assessment of chemicals when simulating real exposure scenarios; simultaneous multi-chemicals' low dose exposure. Food Chem Toxicol 96: 174-176, 2016.

8. Tsatsakis AM, Kouretas D, Tzatzarakis MN, Stivaktakis P, Tsarouhas K, Golokhvast KS, Rakitskii VN, Tutelyan VA, Hernandez AF, Rezaee R, et al: Simulating real-life exposures to uncover possible risks to human health: A proposed consensus for a novel methodological approach. Hum Exp Toxicol 36: 554-564, 2017.

9. Kostoff RN, Goumenou M and Tsatsakis A: The role of toxic stimuli combinations in determining safe exposure limits. Toxicol Rep 5: 1169-1172, 2018.

10. Docea AO, Calina D, Goumenou M, Neagu M, Gofita E and Tsatsakis A: Study design for the determination of toxicity from long-term-low-dose exposure to complex mixtures of pesticides, food additives and lifestyle products. Toxicol Lett 258: S179, 2016.

11. Docea AO, Gofita E, Goumenou M, Calina D, Rogoveanu O, Varut M, Olaru C, Kerasioti E, Fountoucidou P, Taitzoglou I, et al: Six months exposure to a real life mixture of 13 chemicals' below individual NOAELs induced non monotonic sex-dependent biochemical and redox status changes in rats. Food Chem Toxicol 115: 470-481, 2018

12. Docea AO, Goumenou M, Calina D, Arsene AL, Dragoi CM, Gofita E, Pisoschi CG, Zlatian O, Stivaktakis PD, Nikolouzakis TK, et al: Adverse and hormetic effects in rats exposed for 12 months to low dose mixture of 13 chemicals: RLRS part III. Toxicol Lett 310: 70-91, 2019.

13. Renieri EA, Goumenou M, Kardonsky DA, Veselov VV, Alegakis AK, Buha A, Tzatzarakis MN, Nosyrev AE, Rakitskii VN, Kentouri M, et al: Indicator PCBs in farmed and wild fish in Greece - Risk assessment for the Greek population. Food Chem Toxicol 127: 260-269, 2019.

14. Mesnage R, Antoniou MN, Tsoukalas D, Goulielmos GN and Tsatsakis A: Gut microbiome metagenomics to understand how xenobiotics impact human health. Curr Opin Toxicol 11-12: $51-58,2018$

15. Tsiaoussis J, Antoniou MN, Koliarakis I, Mesnage R, Vardavas CI, Izotov BN, Psaroulaki A and Tsatsakis A: Effects of single and combined toxic exposures on the gut microbiome: Current knowledge and future directions. Toxicol Lett 312: 72-97, 2019.

16. Espinoza JL: Machine learning for tackling microbiota data and infection complications in immunocompromised patients with cancer. J Intern Med 284: 189-192, 2018.

17. Ribeiro E, Ladeira C and Viegas S: EDCs Mixtures: A Stealthy Hazard for Human Health? Toxics 5: E5, 2017.

18. Street ME, Angelini S, Bernasconi S, Burgio E, Cassio A, Catellani C, Cirillo F, Deodati A, Fabbrizi E, Fanos V, et al: Current knowledge on endocrine disrupting chemicals (EDCs) from animal biology to humans, from pregnancy to adulthood: Highlights from a national italian meeting. Int J Mol Sci 19: E1647, 2018

19. Munn S and Goumenou M: Thresholds for Endocrine Disrupters and Related Uncertainties. Report of the Endocrine Disrupters Expert Advisory Group. Publications Office of the European Union, 2013. https://publications.europa.eu/en/publicationdetail/-/publication/a1a68b31-c013-484a-b4f0-ce089822aad8/ language-en. Accessed July 11, 2014. 
20. Day P, Green RM, Gross M, Weltje L and Wheeler JR: Endocrine Disruption: Current approaches for regulatory testing and assessment of plant protection products are fit for purpose. Toxicol Lett 296: 10-22, 2018.

21. International Programme on Chemical Safety: Global assessment on the state of the science of endocrine disruptors. World Health Organization, Geneva, 2002. https://apps.who.int/ iris/handle/10665/67357.

22. Munn S and Goumenou M: Key scientific issues relevant to the identification and characterisation of endocrine disrupting substances. Report of the Endocrine Disrupters Expert Advisory Group. Publications Office of the European Union 2013. https://publications.europa.eu/en/publication-detail/-/ publication/4b84ccc2-422d-4bd1-97da-1f414ad52c27/languageen. Accessed April 2, 2013.

23. European Chemicals Agency (ECHA) and European Food Safety Authority (EFSA) with support from the Joint Research Centre (JRC): Guidance for the identification of endocrine disruptors in the context of Regulations (EU) No 528/2012 and (EC) No 1107/2009. EFSA J 16: e05311, 2018.

24. Schug TT, Johnson AF, Birnbaum LS, Colborn T, Guillette LJ Jr, Crews DP, Collins T, Soto AM, Vom SaalFS, McLachlan JA, et al: Minireview: Endocrine Disruptors: Past Lessons and Future Directions. Mol Endocrinol 30: 833-847, 2016.

25. Darbre PD: Endocrine Disruptors and Obesity. Curr Obes Rep 6 : $18-27,2017$.

26. Ravichandran G, Lakshmanan DK, Raju K, Elangovan A, Nambirajan G, Devanesan AA and Thilagar S: Food advanced glycation end products as potential endocrine disruptors: An emerging threat to contemporary and future generation. Environ Int 123: 486-500, 2019.

27. Lee YM, Jacobs DR Jr and Lee DH: Persistent Organic Pollutants and Type 2 Diabetes: A Critical Review of Review Articles. Front Endocrinol (Lausanne) 9: 712, 2018

28. Lind PM and Lind L: Endocrine-disrupting chemicals and risk of diabetes: An evidence-based review. Diabetologia 61: 1495-1502, 2018.

29. Idowu O, Semple KT, Ramadass K, O'Connor W, Hansbro P and Thavamani P: Beyond the obvious: Environmental health implications of polar polycyclic aromatic hydrocarbons. Environ Int 123: 543-557, 2019.

30. Di Nisio A and Foresta C: Water and soil pollution as determinant of water and food quality/contamination and its impact on male fertility. Reprod Biol Endocrinol 17: 4, 2019.

31. Rahmani S, Pour Khalili N, Khan F, Hassani S, Ghafour-Boroujerdi E, Abdollahi $M$ and Bisphenol $A$ : Bisphenol A: What lies beneath its induced diabetes and the epigenetic modulation? Life Sci 214: 136-144, 2018.

32. Petrakis D, Vassilopoulou L, Mamoulakis C, Psycharakis C, Anifantaki A, Sifakis S, Docea AO, Tsiaoussis J, Makrigiannakis A and Tsatsakis AM: Endocrine Disruptors Leading to Obesity and Related Diseases. Int J Environ Res Public Health 14: 1282, 2017.

33. Trasande L, Zoeller RT, Hass U, Kortenkamp A, Grandjean P, Myers JP, DiGangi J, Bellanger M, Hauser R, Legler J, et al: Estimating burden and disease costs of exposure to endocrine-disrupting chemicals in the European union. J Clin Endocrinol Metab 100: 1245-1255, 2015.

34. Attina TM,HauserR, SathyanarayanaS,Hunt PA,Bourguignon JP, Myers JP, DiGangi J, Zoeller RT and Trasande L: Exposure to endocrine-disrupting chemicals in the USA: A population-based disease burden and cost analysis. Lancet Diabetes Endocrinol 4 996-1003, 2016

35. De Coster S and van Larebeke N: Endocrine-disrupting chemicals: Associated disorders and mechanisms of action. J Environ Public Health 2012: 713696, 2012.

36. Luechtefeld T, Marsh D, Rowlands $C$ and Hartung T: Machine Learning of Toxicological Big Data Enables Read-Across Structure Activity Relationships (RASAR) Outperforming Animal Test Reproducibility. Toxicol Sci 165: 198-212, 2018.

37. Ding D, Xu L, Fang H, Hong H, Perkins R, Harris S, Bearden ED, Shi L and Tong W: The EDKB: An established knowledge base for endocrine disrupting chemicals. BMC Bioinformatics 11 (Suppl 6): S5, 2010.

38. Castello P, Wittwehr C and Goumenou MP: Endocrine Active Substances Information System (EASIS) Software Implementation Plan. Technical Report. EU PUBSY No. JRC76923, 2012.
39. OECD: Revised Guidance Document 150 on Standardised Test Guidelines for Evaluating Chemicals forEndocrine Disruption, OECD Series on Testing and Assessment. OECD Publishing, Paris, 2018. https://doi.org/10.1787/9789264304741-en.

40. Browne P, Noyes PD, Casey WM and Dix DJ: Application of adverse outcome pathways to U.S. EPA's endocrine disruptor screening program. Environ Health Perspect 125: 096001, 2017.

41. Mesnage R, Biserni M, Genkova D, Wesolowski L and Antoniou MN: Evaluation of neonicotinoid insecticides for oestrogenic, thyroidogenic and adipogenic activity reveals imidacloprid causes lipid accumulation. J Appl Toxicol 38: 1483-1491, 2018

42. Mesnage R, Phedonos A, Arno M, Balu S, Corton JC and Antoniou MN: Editor's Highlight: Transcriptome Profiling Reveals Bisphenol A Alternatives Activate Estrogen Receptor Alpha in Human Breast Cancer Cells. Toxicol Sci 158: 431-443, 2017.

43. Raies AB and Bajic VB: In silico toxicology: Computational methods for the prediction of chemical toxicity. Wiley Interdiscip Rev Comput Mol Sci 6: 147-172, 2016.

44. Delfosse V, Dendele B, Huet T, Grimaldi M, Boulahtouf A, Gerbal-Chaloin S, Beucher B, Roecklin D, Muller C, Rahmani R, et al: Synergistic activation of human pregnane $\mathrm{X}$ receptor by binary cocktails of pharmaceutical and environmental compounds. Nat Commun 6: 8089, 2015.

45. Sarigiannis DA and Gotti A: Biology-based dose-response models for health risk assessment of chemical mixtures. Fresenius Environ Bull 17: 1439-1451, 2008.

46. Vuorinen A, Odermatt A and Schuster D: In silico methods in the discovery of endocrine disrupting chemicals. J Steroid Biochem Mol Biol 137: 18-26, 2013.

47. Wahl J and Smieško M: Endocrine Disruption at the Androgen Receptor: Employing Molecular Dynamics and Docking for Improved Virtual Screening and Toxicity Prediction. Int J Mol Sci 19: 1784, 2018

48. Kar S, Ghosh S and Leszczynski J: Single or mixture halogenated chemicals? Risk assessment and developmental toxicity prediction on zebrafish embryos based on weighted descriptors approach. Chemosphere 210: 588-596, 2018.

49. Mansouri K, Abdelaziz A, Rybacka A, Roncaglioni A, Tropsha A, Varnek A, Zakharov A, Worth A, Richard AM, Grulke CM, et al: CERAPP: Collaborative Estrogen Receptor Activity Prediction Project. Environ Health Perspect 124: 1023-1033, 2016.

50. Kaserer T, Beck RK, Akram M, Odermatt A and Schuster D: Pharmacophore Models and Pharmacophore-Based Virtual Screening: Concepts and Applications Exemplified on Hydroxysteroid Dehydrogenases. Molecules 20: 22799-22832, 2015.

51. Ruiz P, Sack A, Wampole M, Bobst S and Vracko M: Integration of in silico methods and computational systems biology to explore endocrine-disrupting chemical binding with nuclear hormone receptors. Chemosphere 178: 99-109, 2017.

52. Robinson-Rechavi M, Carpentier A-S, Duffraisse $M$ and Laudet V: How many nuclear hormone receptors are there in the human genome? Trends Genet 17: 554-556, 2001

53. Wang Z, Liu H and Liu S: Low-Dose Bisphenol A Exposure: A Seemingly Instigating Carcinogenic Effect on Breast Cancer. Adv Sci (Weinh) 4: 1600248, 2016.

54. Nohynek GJ, Borgert CJ, Dietrich D and Rozman KK: Endocrine disruption: Fact or urban legend? Toxicol Lett 223: 295-305, 2013.

55. Schneider S, Fussell KC, Melching-Kollmuss S, Buesen R, Gröters S, Strauss V, Jiang $X$ and van Ravenzwaay B: Investigations on the dose-response relationship of combined exposure to low doses of three anti-androgens in Wistar rats. Arch Toxicol 91: 3961-3989, 2017.

56. Roszko MŁ, Kamińska M, Szymczyk K, Piasecka-Jóźwiak K and Chabłowska B: Endocrine disrupting potency of organic pollutant mixtures isolated from commercial fish oil evaluated in yeast-based bioassays. PLoS One 13: e0197907, 2018.

57. Seeger B, Klawonn F, Nguema Bekale B and Steinberg P: Mixture Effects of Estrogenic Pesticides at the Human Estrogen Receptor $\alpha$ and $\beta$. PLoS One 11: e0147490, 2016.

58. Vandenberg LN, Colborn T, Hayes TB, Heindel JJ, Jacobs DR Jr, Lee DH, Shioda T, Soto AM, vom Saal FS, Welshons WV, et al: Hormones and endocrine-disrupting chemicals: Low-dose effects and nonmonotonic dose responses. Endocr Rev 33: $378-455,2012$

59. Sarigiannis D, Gotti A, Cimino Reale G and Marafante E: Reflections on new directions for risk assessment of environmental chemical mixtures. Int J Risk Assess Manag 13: 216-241, 2009. 
60. Bliss CI: The calculation of the dosage-mortality curve. Ann Appl Biol 22: 134-167, 1935.

61. Sarigiannis DAHU and Hansen U: Considering the cumulative risk of mixtures of chemicals - a challenge for policy makers. Environ Health 11 (Suppl 1): S18, 2012.

62. Goumenou M and Tsatsakis A: Proposing new approaches for the risk characterisation of single chemicals and chemical mixtures: The source related Hazard Quotient (HQS) and Hazard Index (HIS) and the adversity specific Hazard Index (HIA). Toxicol Rep 6: 632-636, 2019.
63. Katsikantami I, Colosio C, Alegakis A, Tzatzarakis MN, Vakonaki E, Rizos AK, Sarigiannis DA and Tsatsakis AM: Estimation of daily intake and risk assessment of organophosphorus pesticides based on biomonitoring data - The internal exposure approach. Food Chem Toxicol 123: 57-71, 2019.

64. Papadakis GZ, Karantanas AH, Tsiknakis M, Tsatsakis A, Spandidos DA and Marias K: Deep learning opens new horizons in personalized medicine. Biomed Rep 10: 215-217, 2019.

(i) (i) $($ This work is licensed under a Creative Commons Attribution-NonCommercial-NoDerivatives 4.0 International (CC BY-NC-ND 4.0) License. 